\author{
„PLAGIATE BEI DIPLOM- UND DOKTORARBEITEN WERDEN IN DEN \\ INGENIEUR- UND NATURWISSENSCHAFTEN DURCH DIE ENGE \\ ZUSAMMENARBEIT MIT DEM BETREUER VERHINDERT. WIR MÜSSEN \\ JEDOCH DARAUF ACHTEN, DASS BEI DEN ARBEITEN SEHR SORGFÄLTIG \\ ZITIERT WIRD.“
}

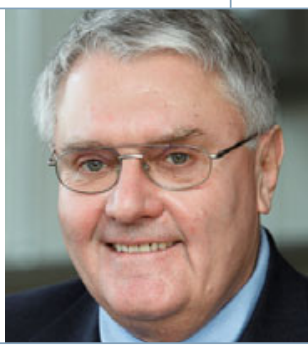

Wolfgang E. Trommer

\title{
Plagiate - auch in den Naturwissenschaften?
}

Am 12. April des Jahres hat der Deutsche Hochschulverband (DHV) auf seiner Delegiertenversammlung in Potsdam ohne Gegenstimmen eine Resolution „Wider die Plagiate“ verabschiedet. Kernaussagen betreffen die Aufforderung an alle „Wissenschaftlerinnen und Wissenschaftler sowie die Prüfungsämter, geeignete Maßnahmen zu ergreifen, um die eigenständige wissenschaftliche Leistung von studentischen Seminar- und Abschlussarbeiten überprüfen zu können. Alle akademischen Qualifikationsarbeiten sind demnach auch digital einzureichen, um besser überprüfen zu können, ob es Übereinstimmungen mit fremden Texten gibt“. Dies gilt natürlich auch und insbesondere für Dissertationen. Dass eine Erklärung abgegeben werden muss, wonach die Arbeit selbstständig und ohne Hilfeleistungen Dritter angefertigt worden ist, bzw. nur mit den angegebenen Hilfen, ist selbstverständlich. Nicht aber die oft damit in den Prüfungsordnungen verbundene eidesstattliche Erklärung. Nur in drei Bundesländern, Nordrhein-Westfalen, Bayern und dem Saarland, sind Universitäten ermächtigt worden, diese rechtlich relevant entgegenzunehmen. Die Entscheidung, dies auch in den Prüfungsordnungen zu verankern, liegt aber bei den Hochschulen und wird von Standort zu Standort unterschiedlich gehandhabt. Es gilt also, dieses Recht nicht nur auf alle Bundesländer auszudehnen, sondern die Hochschulen auch aufzufordern, es anzuwenden. Nur so ergeben sich aus dem möglichen Meineid strafrechtliche Konsequenzen. Zum Glück für Herrn zu Guttenberg hatte die Universität Bayreuth auf eine eidesstattliche Erklärung zugunsten einer „Ehrenerklärung“ verzichtet.

Es gibt noch ein weiteres Problem: die Diplomarbeitsbörsen und ähnliche Sammlungen akademischer Arbeiten. Auch hier zitiere ich aus der Resolution des DHV: „Obwohl der begründete Verdacht besteht, dass ein Teil der von diesen Börsen verkauf- ten Arbeiten für Plagiate verwendet wird, gibt es keine rechtliche Handhabe, gegen dieses Geschäftsmodell vorzugehen“. Und weiter aus der Resolution, ,alle Hochschulen werden aufgefordert, in den ersten Semestern die Studierenden in die Kultur wissenschaftlichen Arbeitens einschließlich der fachspezifischen Besonderheiten einzuführen“. Aber eben diese fachspezifischen Besonderheiten führen $\mathrm{zu}$ den Problemen in experimentellen Fächern.

Diplom- und Doktorarbeiten in den Ingenieur- und Naturwissenschaften entstehen fast immer in enger Zusammenarbeit mit dem Betreuer; externe Arbeiten sind eher die Ausnahme. Wöchentliche bis sogar tägliche Besprechung der experimentellen wissenschaftlichen Ergebnisse ist die Regel. Das kann zwar wissenschaftlichen Betrug, das heißt die Manipulation und Fälschung von Daten, nicht sicher verhindern, wohl aber Plagiate, wenn der Betreuer nicht völlig versagt. Ist es aber wirklich so einfach? Zur Erzielung der wissenschaftlichen Ergebnisse werden oft Apparaturen genutzt, die schon vor Jahren aufgebaut worden sind und an denen danach mehrere Doktoranden Messungen durchgeführt haben. Es ist nicht immer sinnvoll, das Rad stets aufs Neue zu erfinden, das heißt, die (gängige) Messapparatur mit eigenen Worten zu beschreiben. Gleiches gilt für Standardversuchsprotokolle. Natürlich müsste auch hier die Vorgängerarbeit zitiert werden, aber nicht unbedingt in jedem Absatz und schon gar nicht in Anführungszeichen.

Was aber ist, wenn ein Absolvent später einmal ein öffentliches Amt bekleidet und, wie es Der Spiegel im Heft 29 dieses Jahres formuliert hat, im Schwarm auf ihn Jagd gemacht wird. „Sie sind anonym, sie treffen sich im Internet, sie scannen Dissertationen und überführen Politiker als Fälscher“. Dann ist es zu spät, darauf hinzuweisen, dass dieser Teil der Dissertation wissenschaftlich nicht relevant ist. Es würde damit vermutlich nur das
Gegenteil erreicht; wer sich verteidigt, gesteht die Schuld ein, oder zumindest hält es den „Fall“ noch länger in den Medien.

Erwähnt seien noch zwei Aspekte: Es gibt Programme zur Erkennung von Plagiaten, die nach Eingabe des Textes selbständig im Internet nach übereinstimmenden Passagen suchen. Diese haben jedoch sehr hohe Lizenzgebühren (z. B. Turnitin) - zu hoch für den persönlichen Gebrauch, insbesondere für Studierende, die aus Gründen der Fairness Zugang zu diesen Programmen haben sollten. Außerdem greifen manche Plagiat-Erkennungsprogramme (z. B. SafeAssign) auf einen Server in den USA zu, auf den Examensarbeiten hochgeladen werden müssen; eine vorab-Veröffentlichung in dieser Form ist besonders dann kritisch, wenn patentrechtlich relevante Ergebnisse erzielt worden sind oder auch wissenschaftlich hochkompetitive Forschungsbereiche berührt sind.

Fazit: Wir müssen darauf achten, dass auch in diesen Teilen einer Diplom- oder Doktorarbeit sehr sorgfältig zitiert wird, auch dann, wenn darunter die Lesbarkeit leidet.

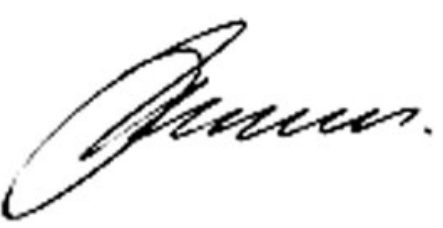

Wolfgang E. Trommer, Technische Universität Kaiserslautern

Korrespondenzadresse:

Prof. Dr. Wolfgang E. Trommer Fachbereich Chemie

Technische Universität Kaiserslautern

Erwin-Schrödinger-Straße 52

D-67653 Kaiserslautern

Tel.: 0631-205-2045

Fax: 0631-205-3419

trommer@chemie.uni-kl.de 